\title{
A qualitative study of illness identity: schizophrenia and depression
}

\author{
Namino Ottewell \\ School of Social Sciences, Waseda University, Tokyo, Japan
}

\begin{abstract}
Illness identity refers to the interpretation of having mental illness in persons who regard themselves as mentally ill. Illness identity can impact people diagnosed with mental illness negatively. However, little is known about the background of illness identity. This study aimed to investigate the process whereby mental health service users accept their diagnosis and develop an illness identity and to compare the processes between schizophrenia and depression. Twenty persons with schizophrenia and depression were interviewed, and the data were analysed using grounded theory approach. The major findings were as follows: i) while those with schizophrenia were shocked by their diagnosis and took time to accept it, this was not true for those with depression; ii) for participants with depression, it was relatively easy to accept their diagnosis, as they thought that depression was a normal illness; participants with schizophrenia needed to learn about their illness, know the efficacy of psychiatric medication and reduce their prejudice against mental illness to accept their diagnosis; and iii) participants' illness identity encompassed sickness and normality with sickness being related to taking psychiatric medication and normality being associated with having regular work, acting as other people do or living in the community. These findings suggest the influence of social attitudes to each illness on illness identity and the complex nature of illness identity.
\end{abstract}

\section{Introduction}

Yanos, Roe and Lysaker ${ }^{1}$ proposed a concept termed 'illness identity' based on the research findings on the influence of the experience and diagnosis of mental illness. Illness identity refers to "the set of roles and attitudes that a person has developed about him or herself in relation to his or her understanding of mental illness' (p. 74). Thus, it is proposed that individuals do not merely experience symptoms of mental illness but also interpret their experience of the illness. This concept is applied to cases in which an individual has decided to characterise his/her experiences, which might be regarded as psychiatric symptoms by a mental health professional, as at least

\footnotetext{
Correspondence: Namino Ottewell, School of Social Sciences, Waseda University 1-6-1, Nishiwaseda, Shinjyuku-ku, Tokyo 1698050, Japan.

Tel.: +81.3.3208.0545.

E-mail: ottewelln@ml1.net

Key words: Illness identity; Mental illness; Depression; Schizophrenia.

Conflict of interest: the author declares no potential conflict of interest.

Funding: none.

Received for publication: 16 March 2018.

Revision received: 9 August 2018.

Accepted for publication: 9 August 2018.

This work is licensed under a Creative Commons Attribution NonCommercial 4.0 License (CC BY-NC 4.0).

C Copyright N. Ottewell, 2018

Licensee PAGEPress, Italy

Qualitative Research in Medicine \& Healthcare 2018; 2:84-93

doi:10.4081/qrmh.2018.7420
}

partly caused by mental illness. As such, the concept is not applied to cases in which an individual has experiences which might be described as symptoms of mental illness but does not consider himself / herself ill. The concept of 'illness identity' was proposed as an alternative to earlier concepts such as 'engulfment'. ${ }^{2}$ Whilst 'engulfment' refers to a person's self-concept and behaviour becoming increasingly organised around the psychiatric patient role, 'illness identity' allows for the multiple ways in which persons might make sense of having mental illness. Thus, 'illness identity' includes, for example, interpretations of having mental illness which do not seem to fit the patient role, such as 'empowered' identities. ${ }^{3,4}$

In the present study, as in the study by Yanos et al., ${ }^{1}$ 'identity' refers to i) the social categories which an individual uses to describe himself/herself and ii) the social categories which others use to describe the individual. ${ }^{5}$ It has been well documented that psychiatric diagnosis and experiences of mental illness can significantly influence one's identity. ${ }^{6}$ A meta-synthesis of qualitative studies on the experience of psychosis revealed that whereas people experienced losses of self-esteem, self-identity and the sense of having a coherent self at the early stage of psychosis, once recovery was underway, they regained a sense of having a self and rebuilt identity and self-esteem, with the ability to regain identity by asserting a unique individual identity. ${ }^{7}$

Reviewing the existing empirical literature, Yanos et $a l .{ }^{1}$ revealed the impact of illness identity on the course and recovery from severe mental illness; illness identity can be detrimental to recovery and impacts one's hope and self-esteem negatively. However, although a large body of study has investigated the impact of illness identity on people with mental health problems, little is known 
about the process whereby illness identity develops. It is important to investigate the process, because by doing that, it is possible for us to clarify the background of illness identity. Furthermore, understanding the process will also provide us with a more comprehensive picture of mental illness experience and influences of mental illness and psychiatric diagnosis on one's identity.

The present study, therefore, aimed to examine the process whereby people with psychiatric diagnosis develop illness identity. In doing this, the present study also investigated how they accepted their psychiatric diagnosis, since it is necessary for one to accept that they have a mental illness in order to develop illness identity. Acceptance was defined as an individual coming to consider that their somatic and/or psychological changes have been caused by mental health problems. While mental illness includes various categories, this study focussed upon two specific illnesses, schizophrenia and depression. This is firstly because these two illnesses are perceived differently by lay people, with schizophrenia being more stigmatised. ${ }^{8,9}$ The second reason is that the studies on the impact of psychiatric diagnosis and experiences of mental illness on one's identity have mainly focussed on people with psychosis, and the impact on the identity of people with depression has been relatively unexamined. By comparing the process whereby persons diagnosed with schizophrenia develop their illness identity with that of persons with depression, this study analyses the affinities and differences in the processes whereby they develop their illness identity between these two illnesses.

\section{Materials and Methods}

\section{Participants}

The data for this study were derived from 20 interviews conducted primarily to explore the experiences with stigma among people with mental illness. After obtaining the university's ethical approval, I recruited participants from community activity support centres for psychiatric patients and psychiatric hospitals in Tokyo, Japan.

The inclusion criteria were as follows: i) people diagnosed with schizophrenia or depression by health professionals; ii) people over the age of 20 years.

The following exclusion criteria were applied: i) people experiencing acute symptoms of mental illness; ii) people experiencing dementia, intellectual disability or substance abuse; iii) people having problems with interactions with an interviewer.

A total of 20 people, including 10 men and 10 women, participated in this study, twelve of whom had been diagnosed with schizophrenia and eight with depression. The average duration of treatment was 14 years (ranging from 1-40 years). The age and employment status of participants are presented in Table 1.

\section{Procedure}

Interviews were semi-structured and face-to-face, and each interview lasted between 25 and 50 minutes. While the majority of the questions were related to stigma against mental illness, participants were also asked about their feelings and thoughts concerning their psychiatric diagnosis. The questions regarding their feelings and thoughts about diagnosis included the following: i) When and how were you first diagnosed with mental illness? ii) How do (or did) you feel about your diagnosis? iii) If you were not told your diagnosis by your doctor initially, when and how did you learn about your diagnosis and how did you feel about it? iv) Do you find it easy or difficult to mention your diagnosis to others? v) Do you think if your diagnosis were different (for example, if it were not schizophrenia but depression), it would be easier to disclose it to others? and vi) Do you accept your diagnosis? If so,

Table 1. Age and employment status of participants.

\begin{tabular}{lll}
\hline Age & $20-25$ & 1 \\
\hline & $26-34$ & 11 \\
\hline $35-44$ & 5 \\
\hline Employment status & $45-54$ & 1 \\
\hline & $55+$ & 2 \\
\hline Job training & 5 \\
\hline Sheltered job & 1 \\
\hline Special employment positions for people with disabilities & 4 \\
\hline Employed (full time) & 5 \\
\hline Employed (part time) & 1 \\
\hline Looking after home and family & 1 \\
\hline
\end{tabular}


how did you accept it? Participants were also asked to talk about their experiences of receiving and accepting psychiatric diagnosis freely. All participants provided a written informed consent form prior to an interview and agreed to their interviews being recorded.

\section{Data analysis}

I analysed interview data by employing the grounded theory approach. ${ }^{10}$ I transcribed the recording of each interview verbatim, and divided the transcripts into segments using natural breaks as cut-off points to code them. Codes and categories were reviewed as the results of the analysis were accumulated, following which they were accepted, modified, discarded or replaced with new ones. I examined relationships between categories by comparing them with one interview case, contrasting them across other cases as well as by reviewing analysis memoranda. Clarifying these relationships led to the emergence of a core category, namely 'living with the self that has changed because of the mental illness itself and because of the psychiatric diagnosis label'. In the process of the analysis, comments and advice from three other researchers were reflected in order to increase validity of the analysis.

The interviews were designed primarily to explore stigma experiences among participants, and as such, this core category is mainly related to participants' experiences after they accepted their diagnosis (e.g. how they concealed their condition). However, for the purpose of this study, the sections below focus on how they came to accept their diagnosis and develop an illness identity.

\section{Results}

\section{Receiving a psychiatric diagnosis}

The participants' reports indicated two broad patterns in terms of how they came to be diagnosed as mentally ill. With regards to the first one, participants noticed recurrent changes in themselves, including insomnia, suicidal thoughts and/or hearing voices. They thought that these changes might be caused by mental health problems and sought a diagnosis. The second pattern involved other people (in most cases, participants' families) noticing changes in participants and taking them to a doctor. In this case, even if participants had been aware of the changes, which had been mostly cognitive changes such as auditory hallucinations and delusions, before their first visit to hospital, most of them had not thought of these changes as psychiatric symptoms. In most cases of the second pattern, participants had not been informed of their diagnosis for several years (ranging from 2-11 years), although their families were informed of it. Participants were usually unaware of their diagnosis while receiving psychiatric treatment. Apart from one, these participants first became aware of their diagnosis when they saw their patient record or application forms for benefits. Although they stated that they had been shocked at the discovery of their diagnosis, no participants expressed dissatisfaction with their family for not having told them.

Whilst all eight participants with depression went to hospital of their own free will, the majority with schizophrenia $(66 \%$ or $8 / 12)$ were taken to hospital by other people for the initial consultation. There was a marked contrast in the reaction to their diagnosis between those with depression and those with schizophrenia, with the former generally being convinced and the latter being puzzled or shocked. Two participants were puzzled because they knew nothing about schizophrenia, whereas other participants were shocked because they regarded it as a serious illness or thought it was related to violence. One of the participants (Anna) stated, 'I was shocked when my doctor told me I had schizophrenia, not depression, because I thought that schizophrenia was much worse than depression and incurable'.

All participants, except for three, were reluctant to disclose their diagnosis to others. The majority of participants with schizophrenia felt that it would be easier to disclose their condition if it were not schizophrenia but depression, as they thought that depression was well-regarded among lay people, whereas schizophrenia was not understood properly and related to images of madness or violence. Participants with depression shared this view, stating that they would never disclose their diagnosis if it were not depression but schizophrenia. In terms of the diagnosis of depression, none of the participants with depression considered that their diagnosis itself mattered. For all the participants with depression, except for one, it was the fact that depression was a mental illness which made them consider whether they should disclose it to others, because they were aware that people were often prejudiced against people with mental illness, regardless of their diagnosis.

\section{Acceptance of diagnosis}

At the time of the interviews, all 20 participants believed that they did indeed have a mental illness. Of the participants with depression, four stated that they had selfdiagnosed their condition before seeing a doctor. Thus, receiving a psychiatric diagnosis probably implied a confirmation of their changes. Three participants with depression were not certain of what their diagnosis would be, although all of them did not appear to have been puzzled by it. They often heard the word depression in the media, and more importantly, they agreed with the information about depression which they saw in the media. Thus, they had already accepted that there was indeed an illness called depression even before receiving a formal diagnosis. One participant was not initially convinced by her diagnosis and thought that she had a borderline personality disorder because of her self-harming behaviour; however, she eventually came to think that she had depression. She (Ruri) stated: 
In the initial period when I began receiving treatment, I wondered if I was experiencing a personality disorder or depression. My doctor expressed that depressive symptoms could've been leading to self-injurious behaviour, but as the self-injuries were so frequent, I wanted to reduce them; thus, I asked for medication that would enable me to reduce self-injury. However, my condition didn't improve, and so, it was eventually decided that receiving medication for depression would be better. I began taking SSRI, and it really seemed to suit me. I became mentally stable, and my self-injuries also decreased. Therefore, I thought that since SSRI was effective, it must've been depression.

Her statement indicates that psychiatric medication played an important role in her acceptance of her diagnosis; she thought of the changes she was experiencing to be symptoms of depression, because SSRI, a widely used antidepressant medication, worked for her. Furthermore, in addition to the effectiveness of psychiatric medication, participants' recognition of depression made it easier for them to accept their diagnoses. As mentioned above, participants shared the view that depression did not hold a very negative image in Japan. Takuya, who had been diagnosed with depression, described how mental illnesses were viewed in Tokyo, after commenting on the intense stigma against mental illness in general in the countryside:

In Tokyo, it's a given - it 's not rare. There didn't seem to be that bad of an image regarding things that you frequently hear about like depression and panic disorders. As might be expected this isn't the case with schizophrenia: its image is different. But if a person is suffering from depression or a panic disorder, it's my feeling that people would treat you as normal in Tokyo.

In his statement, Takuya suggests that depression and panic disorders are normal illnesses, particularly in Tokyo. He also situates schizophrenia in a different position from depression and panic disorders. As can be observed, he began the last sentence with the word but and went on to describing the normalization of depression and panic disorders; this indicates his belief that schizophrenia is different from depression and panic disorders in a negative sense, signifying that schizophrenia is not a normal illness. Although what Takuya mentioned is his understanding of the images of mental illnesses in Tokyo and not all over Japan, the differences between schizophrenia and some other well-known mental illnesses, particularly depression, was recognised by most participants; while having depression is considered normal, having schizophrenia is negatively deviant. For some participants, depression felt normal owing to the large number of patients with depression, as the number of patients with mood disorders, including depression, annually has been over one million since 2005 in Japan. ${ }^{11}$ This meant to them that depression was a common illness that anyone could get. Overall, participants found it easier to accept a diagnosis of depression because depression was indeed an illness but a normal illness according to them. Furthermore, the statements of participants reflected a discourse concerning the relationship between depression and stress, which is widely believed in Japan; if an individual is under enormous stress, he/she will get depressed. ${ }^{12}$ When being asked about the possible reasons for their onsets, participants with depression clearly expressed their views that they became depressed because they were under great stress. By doing this, they also implied that they were normal, because anyone could get depression if they were under high stress.

In contrast, participants with schizophrenia, regardless of how they received information on their condition, needed some time to accept it. All of them rejected their diagnosis at first. They did so because, as mentioned in the previous section, they did not know about schizophrenia, or if they did, they rejected their diagnosis because they thought schizophrenia to be a serious illness or related to violence. For participants who did not know about schizophrenia, it did not make sense to be told by health professionals and their families that they were ill. They believed their experiences, such as someone speaking ill of them, to be true and that it was impossible for them to have a mental illness which brings delusions and hallucinations. For participants who did know about schizophrenia, schizophrenia was an illness from which other people suffer. They never thought that they might suffer from it. Their rejection of their diagnosis also reflected their recognition, as mentioned earlier, that schizophrenia was not a normal illness. All participants with schizophrenia eventually accepted that they did have schizophrenia, but even after that, most of them maintained that having schizophrenia was negatively deviant. For them, whereas it was normal to suffer from depressed mood, anxiety or insomnia when under stress, it was far from normal to have delusions and hallucinations under any circumstances.

When accepting their diagnosis, the majority of participants with schizophrenia found it helpful to read books about the subject to convince them that they did indeed have the illness. These people then noticed that what they had been experiencing, e.g. hearing voices, matched the symptoms of schizophrenia, which made them think that they might actually be suffering from it. Kei described his experience below:

When I was first hospitalised, other patients informed me about my illness. As I didn't recognise I was ill, I went to the door and banged on it, saying, 'Get me out of here!' At this point, other patients asked me various things including what I was thinking about. Subsequently, I was told, 'You are schizo- 
phrenic and if you take medication, you'll be cured', and I thought, 'Oh, really, that was what it was'; this explanation gradually became a reality for me. They also lent me books regarding the illness. I read them and thought, 'Oh, this is the type of illness it is.' About three months after hospitalisation, I understood the type of illness I was suffering from.

Kei's statement clearly shows a process whereby he accepted that he had schizophrenia: although he did not understand what was wrong with him at first, he gradually came to believe that he suffered from schizophrenia by listening to other patients' explanations, realising that what they said was right, and studying about the illness in books. This pattern was seen in some other participants with schizophrenia as well. Kei's statement also reveals that other people played a significant role in his characterising his problems as schizophrenia, and that simultaneously, he also regarded his problems as mental illness himself.

Participants with schizophrenia also accepted their diagnosis by realising that psychiatric medication was effective in dealing with their problems. Knowing that psychiatric medication reduced their symptoms, they came to think that what they had been experiencing might have been caused by mental illness. Participants also had the opportunity to contact other people with mental afflictions once they had become patients themselves. Although some participants had been prejudiced against this type of illness, particularly schizophrenia, which made it difficult for them to accept their diagnosis, through these experiences with other patients, they realised that not all psychiatric patients were as violent and unpredictable as they had thought them to be and found that there were many patients who were gentle, sensitive and well-functioning. Thus, participants' perception of people with mental illness changed, which helped to reduce their hesitation or sense of shame in regarding themselves as suffering from one.

\section{Developing an illness identity}

The major characteristic in participants' illness identity was the coexistence of sickness and normality; whilst participants thought that they were ill, they also thought that they were normal. This characteristic was shared between participants with schizophrenia and those with depression. Normality consisted of their illness identity because it was one of their interpretations of having mental illness; in their thoughts, having mental illness did not necessarily mean becoming completely different from other people or losing a normal life.

In terms of their recognition of being ill, it was notable that taking psychiatric medication was strongly related to their illness identity. Participants stated: 'I can't live without psychiatric meds, so I'm ill (Kana, depression)', 'I recognise I'm ill by taking medicine (Momo, schizophre- nia)' and 'I'm so ill that I have to rely on medicine. If I didn't need medicine, I wouldn't have the identification booklet' [which are given to those with mental illness] (Misa, depression). Participants were generally positive about taking psychiatric medication, except one who was ambivalent about it - positive about improvement in symptoms but negative about side effects. The main reason why participants were positive about medication was that they felt that it was helpful in reducing symptoms and that they were not experiencing any troublesome side effects. Although some participants stated that they had suffered from severe side effects such as sursumvergence before, it was no longer the case at the time of interview, since they were getting new medication which was unlikely to cause severe side effects; they had changed their medication with their doctor's agreement. Consequently, all participants could pass for normal unless they themselves revealed that they had been diagnosed with mental illness. None of them looked different from other people.

Some participants were reluctant to take medication at first, because they believed that 'if you took medication, you've admitted you're ill' (Riku, schizophrenia). In their recognition, taking or needing psychiatric medication meant being mentally ill. They were not concerned about the actual effects which psychiatric medication might bring to them physically and psychologically, but they were concerned about the connotations which psychiatric medication carried for them and other people, such as being considered sick and abnormal. Although this is not what participants actually experienced, some participants stated that they knew some other people who took their psychiatric medication at work, while telling their colleagues and bosses that the medication was for physical illness, or who changed their medication to new ones which they did not need to take during the day, because they did not want to do so at work. Participants who hesitated to take medication at first shared this concern that they would be thought of as mentally ill.

However, at the time of interview, all participants were taking medication as suggested by their doctor. In participants' characterisation of their experiences qua symptoms of mental illness, not only the fact of taking psychiatric medication itself but also the recognition of the efficacy of psychiatric medication played an important role. For example, Tatsuya had been diagnosed with schizophrenia but denied having the illness and did not take medication suggested by his doctor at first. Tatsuya did not want to take psychiatric medication because he thought, 'I'm not like those with mental illness'. He thought of psychiatric medication as something which people with mental illness should take. As seen above, the connotations which psychiatric medication carried mattered to him. However, he realised later that the medication worked well in terms of reducing auditory hallucinations when taken properly. As such, he eventually felt that, 'it may be true that I've been ill'. In other 
words, Takuya thought that what other people, including health professionals and his family, had told him was probably right, and that his problems had been caused by schizophrenia, which was treatable with psychiatric medication. Conversely, participants also recognised the efficacy of psychiatric medication when they stopped taking it or reduced their dose, since they experienced a relapse or deterioration of symptoms. Participants with schizophrenia, in particular, were careful about continuing to take medication, as they thought that they would have a relapse otherwise.

Other reasons for their recognition of being ill included having an identification booklet for those with mental illness. In Japan, people with mental illness can apply for an identification booklet if they have difficulties in everyday life or social life because of their illness, with which they can receive preferential treatment such as reduction of taxes and fees for public transport. Participants with the booklet stated, 'I have the booklet, so I'm officially mentally ill' (Kana, depression) or 'When people don't understand I'm mentally ill, I show them the booklet so that they can understand I'm actually ill' (Misa, depression).

Their recognition of being ill was also associated with their viewing themselves as different from other people. Those with schizophrenia tended to use the terms 'normal people', 'the normal' or 'regular people' when they meant people without mental illness, whereas those with depression were more likely to use the phrases 'people not having mental illness' or 'people who have never had mental illness'. For example, Masa, who was diagnosed with schizophrenia, stated:

Earlier, I thought it'd be unimaginable for me to go to work again; however, after going through several interviews, I could find work that could be done even though I had this illness. Now, I'm receiving a salary and also paying taxes. I feel that I may have come closer to living the life of a normal person; this gives me immense happiness.

His statement shows that he regards 'the life of a normal person' as having work, receiving salary and paying taxes. Kae, who was diagnosed with depression, on the other hand, stated:

It's hard for me that my husband doesn't understand that I'm ill, but at the same time, I think it'll be impossible for those who have never had depression to understand that [how being depressed feels like].

In her statement, Kae clearly expresses her recognition of her being ill, and yet she also thinks that maybe nothing can be done about her husband's lack of understanding of her illness, as he has never been depressed. In contrast to Masa, who used the word 'a normal person', Kae uses the word 'those who have never had depression'.

As their descriptions of people without mental illness show, participants with schizophrenia seemed to feel more distant from those without the illness than those with depression. For participants, 'being different from other people' meant being unable to work as other people do or having experiences which other people would not have such as hearing voices or self-harming behaviour.

However, all participants thought that they were also normal while simultaneously thinking they were ill. As stated in 'Acceptance of diagnosis', participants understood depression as a normal illness. As such, participants with depression thought that they had an illness but that it was a normal illness and that they were normal in that sense. For instance, Kaito (depression) stated, 'It's a brain illness, and it's not different from other illnesses. In addition, for participants, a normal life generally meant having regular work. As such, it was understandable that participants with regular work, most of whom were those with depression, regarded them as normal.

In contrast to participants with depression, participants with schizophrenia tended to use the words normal people and regular people to distinguish themselves from those without mental illness. However, participants with schizophrenia also recognised that they were ill but simultaneously normal and they were not different from those without mental illness. Ken (schizophrenia), for example, stated that he did not have an image of himself as quite different from other people. At the time of interview, most participants with schizophrenia did not have regular work. Therefore, they did not think of themselves as normal because they had work, as did many of participants with depression, but they regarded themselves as normal in the sense that they 'act as other people do (not act strangely)' or 'live in the community and lead a life as other people do'. With respect to the factor, 'acting as other people do', the invisibility of their symptoms was key. Participants with schizophrenia, in particular, regarded themselves as normal based on comparisons with their past selves. While they regarded their past selves, who had difficulties leading their everyday lives because of severe symptoms of mental illness, as far from normal, they regarded their present selves, who had recovered to a level that other people could not tell from the participants' appearance or behaviour that they had mental illness, as normal. Participants also compared themselves with people suffering from severe symptoms of mental illness. With the same logic as their comparison between past and present selves, they regarded themselves as normal compared to people having difficulties in leading their everyday lives because of severe symptoms of mental illness. Meanwhile, 'living in the community and leading a life as other people do' meant that participants lived in normal places where other people lived, not in special places such as hospitals, and participated in activities during the day. All the participants lived at home, with their family or alone. These living environments could be one of the reasons for their recognition of being normal. Furthermore, six of participants belonged to the community activity support centre, which they visited on all weekdays, and they 
were not treated as patients there. At the centre, all the people, including the staff, used the word members and not $p a-$ tients. Members were encouraged to think about what they want to do at the centre themselves and the staff paid respect to members' voluntary thoughts and acts. This environment could also have contributed to their thought that they were normal.

As mentioned above, one of the participants' criteria of being normal was having regular employment. While participants without regular employment thought themselves as normal based on some other criteria, this did not mean that they had abandoned hope about obtaining work. All the participants without regular employment at the time of interview thought that they would like to apply for regular employment in future. Some participants intended to apply for a job without disclosing their illness, while others wished to apply for the special employment positions for people with disabilities. In Japan, organisations with 45.5 or more employees (one part-time employee is counted as 0.5 person) are legally obliged to employ people with disabilities, including those with mental illness, as $2.2 \%$ of their workforce. Organisations with this obligation, therefore, recruit people with disabilities. The number of people with mental illnesses who were newly employed in the special employment positions has been rapidly increasing (from 14,555 in 2010 to 41,367 in 2016), ${ }^{13}$ and participants were aware of this. Because of this situation, participants thought that they would eventually be able to have a job in the special employment positions for people with disabilities even if they could not obtain normal work, which meant work which people without mental illness do.

Regardless of their diagnosis, participants' recognition of being normal shared this characteristic: they are normal as long as they are on medication, but they are sick given that they have to take medication to be normal. For participants with schizophrenia, in particular, it was almost obligatory to take medication. However, the obligation did not come from others but from themselves. They thought that they had to take medication to stay normal. In participants' recognition, they were not entirely different from other people except that they had to take medication to be normal. As such, psychiatric medication was related both to participants' recognition of being sick and to their recognition of being normal.

\section{Discussion}

This study examined the process whereby persons with schizophrenia and depression accept their diagnosis and develop illness identity. With respect to acceptance of their diagnosis, participants with depression seemed to have agreed with their diagnosis relatively easily because they understood that depression was a common and treatable illness that people did not have very negative feelings about. Participants also thought that depression was a nor- mal illness, which could have led to their acceptance of their diagnosis and their recognition of being normal. In contrast, participants with schizophrenia found it difficult to accept their diagnosis, since they did not have knowledge about the illness or they had negative perceptions of it. They were also aware that people had distorted views on the affliction. These findings indicate that social attitudes to mental illness affect an individual's thoughts about their condition. In Japan, the old term for schizophrenia 'Seishin Bunretsu Byô' (mind-split-disease) was renamed to 'Tôgô Shitchô Shô' (integration disorder) in 2002, as it was not conceptually appropriate and gave a negative impression. ${ }^{14,15}$ Although research has shown that the new term gave a more positive impression than the old one to the general public, ${ }^{16,17}$ the study findings suggest that negative connotations continue to be attached to schizophrenia. In fact, in the present study, both participants who received their diagnosis before the renaming and after found it difficult to accept. Although this phenomenon is not unique to Japan, ${ }^{18,19}$ considering that the Japanese general public were found to be more prejudiced against schizophrenia than those in other countries, $8,20,21$ it may be more difficult for Japanese people with schizophrenia to come to terms with their diagnosis.

For most participants with schizophrenia, their families were the first people who were informed of their diagnoses. The main reason for this practice could be the psychiatrists' concern that their patients might be shocked by the diagnosis. Indeed, some participants stated that their families had been told by their doctor not to disclose the information for this reason. Although many patients have been informed of their diagnosis of schizophrenia recently, ${ }^{22}$ the practice of not disclosing these results to patients appears to be a common practice in Japan, particularly before the new term was introduced. According to Nishimura, ${ }^{23}$ who conducted a survey on disclosure of psychiatric diagnosis to their patients amongst psychiatrists in Japan, most of the contacted 1,000 psychiatrists refused to participate in the study stating that they 'do not give a diagnosis to patients in our hospital'. Although none of participants complained about the practice, this raises the issue of ethical and human rights which needs to be discussed. However, the problem concerning providing the patient with a diagnosis of schizophrenia is not limited to Japan; research conducted in other countries showed that mental health professionals had concerns for the patient regarding giving a named diagnosis of schizophrenia and found it problematic, unproductive and harmful, ${ }^{24,25}$ indicating that the problem is of universal nature.

Participants thought themselves to be simultaneously sick and normal. Their recognition of being sick was strongly related to their taking psychiatric medication, which meant that they regarded medicine taking as an act of sick people. Furthermore, participants understood their illness in a biological manner, given that they believed that medication could reduce their problems or symptoms. The studies conducted in other cultures also found that 
psychiatric medication implied more severe illness for people diagnosed with mental illness and facilitated or reinforced conceptualisation of one's problems in terms of mental illness. ${ }^{26,27}$ Tucker $^{28}$ notes that biological understanding of mental illness functions as a way to move away from negative perceptions against any unspecified mental abnormality. Whilst this may be true in an aspect, a large body of studies has revealed that this is not always the case. In fact, research has shown that people with a belief of biological aetiology of mental illness showed more stigmatising attitudes to people with mental illness than did people with a belief of psychosocial aetiology of mental illness. ${ }^{29-31}$ Participants' biological understanding of mental illness may reflect the fact that they were receiving psychiatric treatment. However, it is unknown whether they chose this type of treatment because they originally endorsed biological understanding or they have a biological understanding because they received psychiatric treatment. Participants with depression accepted their diagnosis relatively easily probably because they believed that their depression had been caused by a psychosocial factor, namely stress.

Whereas Yanos et al. ${ }^{1}$ note that illness identity can lead to detrimental consequences such as lower self-esteem and loss of hope, the findings of this study show that illness identity does not necessarily lead to negative results. Participants' illness identity did not lead only to negative consequences, because their illness identity encompassed two contradictory-looking elements, normality and sickness. In this sense, study participants were different from the clients of the Programme of Assertive Community Treatment (PACT) programme described in Estroff's ethnographic study. ${ }^{32}$ In the case of PACT clients, they thought themselves as sick or different from other people, and people around them also treated them as sick people. Estroff notes that clients received a direct and indirect message that they were sick and never got well daily, and were simultaneously reminded of their defects and differentness through interactions with people without mental illness. It is possible that participants in this study also daily received messages such as you are sick and you are different from others, given that they were on psychiatric treatment. However, they did not look different from others, and they interacted with those without mental illness such as colleagues, friends and neighbours in their everyday lives. Participants might have been reminded of their own deficits and differentness in comparison with people without mental illness daily in the interaction with them, but they also confirmed their normality in the interactions as they behaved normally, thought normally, and worked normally.

Goffman $^{33}$ argues about the relationships and interactions between the normal and the stigmatised. People diagnosed with mental illness are often stigmatised, ${ }^{34,35}$ and in this sense, study participants can be regarded as the stigmatised. In fact, most of them experienced and per- ceived stigma because of their diagnoses, although this article did not deal with this topic in detail. Goffman ${ }^{33}$ regards stigma as 'an attribute that is deeply discrediting' (p. 4), but what is really needed for stigmatisation is not attributes, but language of relationships; someone can be stigmatised in social interactions and attributes in themselves do not cause stigmatisation. Participants were aware of the discredited nature of mental illness, particularly schizophrenia, while they were also aware that depression was less discredited and even regarded as a normal illness. Their awareness partly explains the rejection of diagnosis of participants with schizophrenia and acceptance of diagnosis of participants with depression. Participants ensured that they appeared normal in their everyday lives in order to avoid being stigmatised, and they knew that it was important for them to appear normal. This was one of the reasons why they adhered to medication. Although it may sound contradictory, participants took medication to appear normal and to not be stigmatised, while being aware the negative connotations of psychiatric medication and how others could stigmatise them for taking medication.

Participants' recognition of 'being sick' was both selflabelled and labelled by others. Thoits ${ }^{36}$ argues that people are likely to label themselves as mentally unwell and seek professional help when they experience non-normative feelings which are persistent or recurrent and when they lack social support. Others can help them think that it is not unusual to have those feelings by confirming that said feelings have understandable origins in objective conditions. However, she also notes that others can label people experiencing non-normative feelings as mentally unwell. Thoits' theory seems to be largely applicable to the experience of participants with depression. On the one hand, they thought that they were ill because of recurrent changes in themselves, including insomnia and suicidal thoughts. On the other hand, they thought that it was normal for them to have depression because they were under great stress, although this was rather influenced by the media than by those around them. Labelling by others, in contrast, is largely applicable to the experiences of participants with schizophrenia. In Scheff's theory, ${ }^{37}$ when an individual's deviance becomes a public issue, both this individual and those around him/her try to deal with the situation using stereotypes of mental illness, which they have learnt in everyday life since childhood, as a guide. When other people react to the deviance of this individual in this way, his/her unstructured rule-breaking tends to crystallise in conformity to these stereotypes. In the case of participants with schizophrenia, most were taken to a hospital by other people for their initial consultation, as other people thought the participants to be mentally ill because of their problems such as auditory hallucinations and delusions. These participants were also influenced by other people's views in accepting that they were ill. However, their 'unstructured rule-breaking' did not crystallise, as Scheff argues, but they learned 
to appear normal. This can be partly explained the facts that they lived in normal environments and most others around them, including the staff of the community activity support centre, did not treat them as sick people.

The present study defined acceptance of diagnosis as the process where an individual comes to consider that their somatic and/or psychological changes have been caused by mental health problems, which is similar to the idea of insight. However, research has indicated problems in this idea. Tucker ${ }^{28}$ indicates that the idea of insight should be more diverse, as acceptance of a diagnosis is a complex process where individuals interpret mental illness and reconstruct the category. Hamilton and Roper ${ }^{38}$ criticise the notion of insight, stating that it reduces the dynamic of opinions to a contest between individual and professional opinion. Furthermore, insight is problematic not only as a concept but from the perspective that recognising oneself as mentally ill can also lead to negative consequences such as self-stigma and lower satisfaction with life..$^{39}$ The literature suggests that it is important to develop an identity as a person, not as a patient, when thinking about recovery. ${ }^{40}$ However, the findings of this study suggest that having insight or recognising oneself as mentally ill is not necessarily detrimental to those diagnosed with mental illness. This is in accordance with the findings of prior studies which showed that some individuals had expressed relief at having been given a diagnosis. ${ }^{19,41}$ In this study, participants thought that having mental illness did not necessarily mean becoming completely different from other people or losing a normal life, and they regarded themselves as normal, while simultaneously thinking of themselves as ill. Although it is true that some participants with schizophrenia were shocked at their diagnosis and took time to accept it, this was mainly because they were prejudiced against the illness, thinking that, for example, those with schizophrenia were violent. It is also true that most participants concealed their diagnosis from others, not because they wanted to deny having a mental illness but because they wished to avoid being stigmatised. These results suggest that negative connotations of mental illness, namely stigma, rather than recognising themselves as mentally ill, affected participants. In fact, while research has shown that having insight can lead to negative consequences such as low self-esteem and quality of life, ${ }^{42}$ there is also evidence that rather than 'insight' itself impacts people diagnosed with mental illness negatively, other factors such as shame proneness and selfstigma mediate the associations of insight and negative consequences. ${ }^{43-45}$

Another limitation of this study was that the participants were recruited from community activity support centres for psychiatric patients and psychiatric hospitals, which probably led to the exclusion of persons with mental health diagnoses who rejected their diagnoses or who did not wish to be involved with the mental health system. Research has shown that persons with psychiatric diagnoses who do not regard themselves as mentally ill have their own interpretations regarding their issues, including 'having a bad temper not an illness', 46 'family problems' and 'outbursts' ${ }^{27}$ Whilst the findings of the present study contribute to an understanding of illness identity in people who have accepted their psychiatric diagnoses, future research will need to investigate perspectives of people with mental health diagnoses who do not regard themselves as ill, since interpretation of mental illness varies.

\section{Conclusions}

The present study described the process whereby mental health service users accept their diagnosis and develop an illness identity and compared the processes between schizophrenia and depression. One of the significant difference was that while those with schizophrenia were shocked by their diagnosis and took time to accept it, this was not true for those with depression. This difference reflected social images of schizophrenia as negatively deviant illness and depression normal illness. Participants' illness identity encompassed sickness and normality, with sickness being strongly related to taking psychiatric medication and normality being connected to having a regular job, acting as other people do or living in the community. Taking psychiatric medication, however, was related both to participants' recognition of being sick and to their recognition of being normal, in the sense that they thought that they were normal as long as they were on medication. Whilst research on the lives of people diagnosed with mental illness mainly focuses on their sick side, the findings of the present study clearly reveal that recognition of being normal simultaneously exists in them. It should be important for people diagnosed with mental illness to have this recognition, even if it is partial recognition as in the case of the study participants, because this recognition will help them in a variety of ways, including sustaining self-esteem and keeping or expanding social networks. As we know little about the recognition of being normal in people diagnosed with mental illness, future research is needed to explore this topic further.

\section{References}

1. Yanos PT, Roe D, Lysaker PH. The impact of illness identity on recovery from severe mental illness. Am J Psychiatr Rehab 2010;13:73-93.

2. Lally SJ. Does being in here mean there is something wrong with me? Schizophrenia Bull 1989;15:253-65.

3. Connell M, Schweitzer R, King R. Recovery from firstepisode psychosis: a dialogical perspective. Bull Menninger Clin 2015;79:70-90.

4. Robertson E, Lyons A. Living with puerperal psychosis: a qualitative analysis. Psychol Psychother 2003;76:411-31.

5. Thoits PA. Self, identity, stress, and mental health. In: Aneshensel CS, Phelan JC, eds. Handbook of sociology of mental health. New York, NY: Kluwer; 1999. pp 321-344.

6. Estroff SE, Lachicotte WS, Illingworth LC, Johnston A. 
Everybody's got a little mental illness: accounts of illness and self among people with severe, persistent mental illnesses. Med Anthropol Quart 1991;5:331-69.

7. McCarthy-Jones S, Marriott M, Knowles R, et al. What is psychosis? A meta-synthesis of inductive qualitative studies exploring the experience of psychosis. Psychosis 2013;5:1-16.

8. Griffiths KM, Nakane Y, Christensen H, et al. Stigma in response to mental disorders: a comparison of Australia and Japan. BMC Psychiatry 2006;6:21.

9. Pescosolido BA, Martin JK, Long JS, et al. "A disease like any other"? A decade of change in public reactions to schizophrenia, depression, and alcohol dependence. Am J Psychiatry 2010;167:1321-30.

10. Corbin J SA. Basics of qualitative research. 3rd ed. London: Sage; 2008.

11. Ministry of Health LaW. Patient Survey 2014-2015.

12. Kitanaka J. Depression in Japan. Oxford: Princeton University Press; 2012.

13. Employment Security Bureau MoH, Labour and Welfare. The Present State of Employment of People with Disabilities. 2017.

14. Iwadate TUS. Results of questionnaire on the term and concept of schizophrenia. Psychiatr Neurol Japon 1996;98:245-65.

15. Sato M. Renaming schizophrenia: a Japanese perspective. World Psychiatry: Official J World Psychiatr Assoc (WPA) 2006;5:53-5.

16. Koike S, Yamaguchi S, Ojio Y, et al. Long-term effect of a name change for schizophrenia on reducing stigma. Soc Psychiatry Psychiatr Epidemiol 2015;50:1519-26.

17. Takahashi H, Ideno T, Okubo S, et al. Impact of changing the Japanese term for "schizophrenia" for reasons of stereotypical beliefs of schizophrenia in Japanese youth. Schizophrenia Res 2009;112:149-52.

18. Knight MTD, Wykes TIL, Hayward P. 'People don't understand': An investigation of stigma in schizophrenia using Interpretative Phenomenological Analysis (IPA). J Mental Health 2009;12:209-22.

19. Pitt LKM, Welford M, Nothard S, Morrison AP. Impact of a diagnosis of psychosis: user-led qualitative study. Psychiatr Bull 2009;33:419-23.

20. Kurihara T, Kato M, Sakamoto S, et al. Public attitudes towards the mentally ill: a cross-cultural study between Bali and Tokyo. Psychiatry Clin Neurosci 2000;54:547-52.

21. Masuda A, Hayes SC, Twohig MP, et al. Comparing Japanese International College Students' and U.S. College Students' mental-health-related stigmatizing attitudes. J Multicult Counsel Develop 2009;37:178-89.

22. Kako YOR, Shimizu Y. A multicenter study regarding schizophrenia patients' notification of the diagnosis. Psychiatr Neurol Japon 2014;116:813-24.

23. Nishimura, Y. The influence of the term change of schizophrenia on doctor's giving a notice about diagnosis to patients. Jap J Psychiatr Nurs 2004;31:38-42.

24. Moran GS, Oz G, Karnieli-Miller O. Psychiatrists' challenges in considering disclosure of schizophrenia diagnosis in Israel. Qual Health Res 2014;24:1368-80.

25. Outram S, Harris G, Kelly B, et al. Contextual barriers to discussing a schizophrenia diagnosis with patients and families: need for leadership and teamwork training in psychiatry. Acad Psychiatry 2015;39:174-80.

26. Interian A, Martinez IE, Guarnaccia PJ, et al. A qualitative analysis of the perception of stigma among Latinos receiving antidepressants. Psychiatr Serv 2007;58:1591-4.
27. Moses T. Self-labeling and its effects among adolescents diagnosed with mental disorders. Soc Sci Med 2009;68:570-8.

28. Tucker, I. "This is for Life": A discursive analysis of the dilemmas of constructing diagnostic identities. Forum: Qualit Soc Res 2009;10:12.

29. Angermeyer MC, Holzinger A, Carta MG, Schomerus G. Biogenetic explanations and public acceptance of mental illness: systematic review of population studies. Br J Psychiatry 2011;199:367-72.

30. Mojtabai R. Americans' attitudes toward mental health treatment seeking: 1990-2003. Psychiatr Serv 2007;58:642-51.

31. Read J, Haslam N, Sayce L, Davies E. Prejudice and schizophrenia: a review of the 'mental illness is an illness like any other' approach. Acta Psychiatr Scandin 2006;114:303-18.

32. Estroff SE. Making it crazy: an ethnography of psychiatric clients in an american community. California: University of California Press; 1981.

33. Goffman E. Stigma: notes on the management of spoiled identity. Harmondsworth: Penguin; 1963.

34. Lasalvia A, Zoppei S, Van Bortel T, et al. Global pattern of experienced and anticipated discrimination reported by people with major depressive disorder: a cross-sectional survey. Lancet 2013;381:55-62.

35. Thornicroft G, Brohan E, Rose D, et al. Global pattern of experienced and anticipated discrimination against people with schizophrenia: a cross-sectional survey. Lancet 2009;373:408-15.

36. Thoits PA. Self-labeling processes in mental illness: the role of emotional deviance. Am J Sociol 1985;91:221-49.

37. Scheff T. Being mentally ill. Chicago: Aldine; 1965.

38. Hamilton B, Roper C. Troubling 'insight': power and possibilities in mental health care. J Psychiatr Mental Health Nurs 2006;13:416-22.

39. Gaziel M, Hasson-Ohayon I, Morag-Yaffe M, et al. Insight and satisfaction with life among adolescents with mental disorders: assessing associations with self-stigma and parental insight. Eur Psychiatry 2015;30:329-33.

40. Korsbek L. Illness insight and recovery: how important is illness insight in peoples' recovery process? Psychiatr Rehab J 2013;36:222-5.

41. Dinos S, Stevens S, Serfaty M, et al. Stigma: the feelings and experiences of 46 people with mental illness. Qualitative study. Br J Psychiatry 2004;184:176-81.

42. Staring AB, Van der Gaag M, Van den Berge $M$, et al. Stigma moderates the associations of insight with depressed mood, low self-esteem, and low quality of life in patients with schizophrenia spectrum disorders. Schizophrenia Res 2009;115:363-9.

43. Cavelti M, Kvrgic S, Beck EM. Self-stigma and its relationship with insight, demoralization, and clinical outcome among people with schizophrenia spectrum disorders. Comprehens Psychiatry 2012;53:468-79.

44. Lien YJ, Chang HA, Kao YC, et al. Insight, self-stigma and psychosocial outcomes in Schizophrenia: a structural equation modelling approach. Epidemiol Psychiatr Sci 2018;27:176-85.

45. Lien YJ, Chang HA, Kao YC, et al. Self-stigma mediates the impact of insight on current suicide ideation in suicide attempters with schizophrenia: results of a moderated mediation approach. Suicide Life-Threat Behav 2017.

46. Wang JY. Service users' personal experience and interpretation of mental illness: oriental narratives. Int J Soc Psychiatry 2012;58:425-32. 